\title{
CONTRIBUTION TO THE STUDY OF PTERIDOPHYTES OF THE SERRA DO URUBÚ, MARAIAL MUNICIPALITY, PERNAMBUCO STATE, NORTHEASTERN BRAZIL (MARATTIACEAE-VITTARIACEAE)
}

\author{
by
}

IVA CARNEIRO LEÃO BÁRROS, MÁRCIO ROBERTO PIETROBOM-SILVA, GEORGE SIDNEY BARACHO, JOSÉ ALVES DE SIQUEIRA, VANEICIA GOMES DOS SANTOS, ALFREDO MATOS MOURA

Departamento de Botânica, Centro de Ciências Biológicas, Universidade Federal de Pernambuco. Av. Prof. Nelson Chaves, s/n. CEP - 50670 - 420. Cidade Universitária, Recife (Pernambuco, Brasil) (ivaleao@truenet.com.br)

\begin{abstract}
Resumen
Barros, I.C.L, M.R. Pietrobom-Silva, G.S. Baracho, J.A. Siqueira, V.G. Santos \& A.M. Moura (2001). Contribución al estudio de los pteridófitos de la Serra do Urubú, municipio de Maraial, estado de Pernambuco, nordeste de Brasil (Marattiaceae-Vittariaceae). Anales Jard. Bot. Madrid 58(2): 303-310 (en inglés).

En el presente trabajo se estudian los pteridófitos de las familias Marattiaceae, Schizaeaceae, Gleicheniaceae, Cyatheaceae, Pteridaceae y Vittariaceae, colectados en uno de los pocos testimonios de selva fragmentada montana, llamada "floresta atlântica", en la Serra do Urubú, Mata do Ageró, municipio de Maraial, estado de Pernambuco, en el NE de Brasil. Se citan, para las seis familias mencionadas, 22 especies incluidas en 13 géneros, las que preferentemente viven como terrícolas en taludes y barrancos sombríos. Los pliegos están depositados en los herbarios EAN, IPA, UFP y PEUFR.
\end{abstract}

Palabras clave: Pteridophyta, Pernambuco, Brasil, Suramérica.

\begin{abstract}
Barros, I.C.L, M.R. Pietrobom-Silva, G.S. Baracho, J.A. Siqueira, V.G. Santos \& A.M. MOURA (2001). Contribution to the study of pteridophytes of the Serra do Urubú, Maraial municipality, Pernambuco state, Northeastern Brazil (Marattiaceae-Vittariaceae). Anales Jard. Bot. Madrid 58(2): 303-310.

This paper presents a study of the pteridophytes of six families collected in one of the few remaining fragments of atlantic forest in Pernambuco, NE Brazil. 22 species of 13 genera are recorded most of which live on the ground in shady hollows. The specimens are deposited in the EAN, IPA, UFP and PEUFR herbaria.
\end{abstract}

Key words: Pteridophyta, Pernambuco, Brazil, South America.

\section{INTRODUCTION}

The present study is a contribution to the knowledge of the pteridophyte flora of the state of Pernambuco.

The Atlantic Forest in Pernambuco, northeastern Brazil, has largely been destroyed because of the cultivation of sugarcane. What was once an extensive and uninterrupted forest has been reduced to a few fragments, of which $93 \%$ is in stands of about $10 \mathrm{ha}$, and only $7 \%$ is stands of 100 ha or more, which includes one of the larger stands of Atlantic Forest (CONDEPE, 1995). 
The territorial dimensions of the state of Pernambuco provides a diversity of landscapes and vegetation. Data on the flora of Pernambuco are still mainly scarce and restricted to preliminary works and floristic studies such as those of LUETZELBURG (1923), ANDRADE-Lima (1960) and FERrEIRA, RoDAL \& Carvalho (1985). With respect to the pteridophytes of Pernambuco, preliminary floristic contributions were made by PONTUAL (1969, 1971, 1972), BARROS $(1980,1996$, 1997, 1998), Barros \& MARIZ (1990), BARROS \& al. (1988, 1989, 1992a-d, 1997ac), FonSECA (1992), FARIAS \& al. (1992), AMBRósIo (1995), and AMBRósIo \& BARROS (1997).

\section{CHARACTERISTICS OF THE AREA}

The Serra do Urubú is located between $08^{\circ} 42^{\prime} 00^{\prime \prime}$ and $08^{\circ} 43^{\prime} 00^{\prime \prime} \mathrm{S}$, and between $35^{\circ} 48^{\prime} 00^{\prime \prime}$ and $35^{\circ} 49^{\prime} 00^{\prime \prime} \mathrm{W}$, within the municipal districts of Jaqueira, Maraial and Lagoa dos Gatos (fig. 1). The mountain range between 700 and $1.080 \mathrm{~m}$ (VELOSO \& GOES FilHo, 1982). It is dense shady montane forest, and habors one of the few humid forest fragments found in the southern part of Pernambuco. The numerous springs, lakes and ponds, decisive in the maintenance of the vegetation, have been greatly altered by construction of dams that supply the recently created municipal district of Jaqueira. These water sources flow into the Piranji river, one of the main tributaries of the river which crosses several municipal districts.

The complex of mountains formed by the Serra do Urubú and Serra do Espelho embraces several forest fragments with varying degrees of disturbance: Mata do Ageró, Mata da Serra do Quengo, Mata do Caranha e Mata Belarmina. These areas differ in the amount of light that readres their interior, caused by different tree shade which creates various dark and humid microhabitats, altering the soil, relief, and other factors which influence the pteridophyte composition of the communities. The focus of the present study was the forest known as the Mata do Ageró, dominated by trees $20-35 \mathrm{~m}$ tall. This forest presents a wealth of species, particularly epiphytes of the families Araceae, Bromeliaceae and Orchidaceae.

\section{MATERIALS AND METHODS}

We undertook four botanical expeditions and analysed specimens deposited by PEUFR (Herbário Prof. Vasconcelos Sobrinho -Universidade Federal Rural de Pernambuco, Recife, Pernambuco, Brasil) and EAN, IPA, UFP (HoLMGREN \& al., 1990). The species were identified by means of analytic keys (SEHNEM, 1967, 1968, 1970, 1972, 1974, 1978; TRYON \& TRYON, 1982; TRYON \& STOLZE, 1989a, b; LELLINGER, 1987; WINDISCH, 1983, 1992). The ordering of families and genera adopted in this paper follow the proposal of TRYON \& TRYON (1982).

For the habitats naming of the pteridophytes we have follow the classification present in AMBRósIo \& BARROS (1997) and BARROS (1997).

The authors names of taxa have been abbreviated in accordance with patterns presented in BRUMMIT \& POWELL (1992).

\section{RESULTS}

Twenty two species representing thirteen genera and six families were recorded. The data presented reflect collections from near the edges of the highway that passes through the Mata do Ageró, and from slopes within the forest. Further sampling may show better representation of the species found. On the other hand, the low number of species recorded in comparison to data from Mata da Chuva, Bonito municipality, Mata do Engenho Água Azul, Timbaúba municipality, Serra dos Cavalos, Caruarú municipality and Mata do Estado, São Vicente Férrer municipality (Barros, unpublished) may reflect different degrees of sampling in these areas.

A larger number of species was collected on soil, with the greatest number being on 


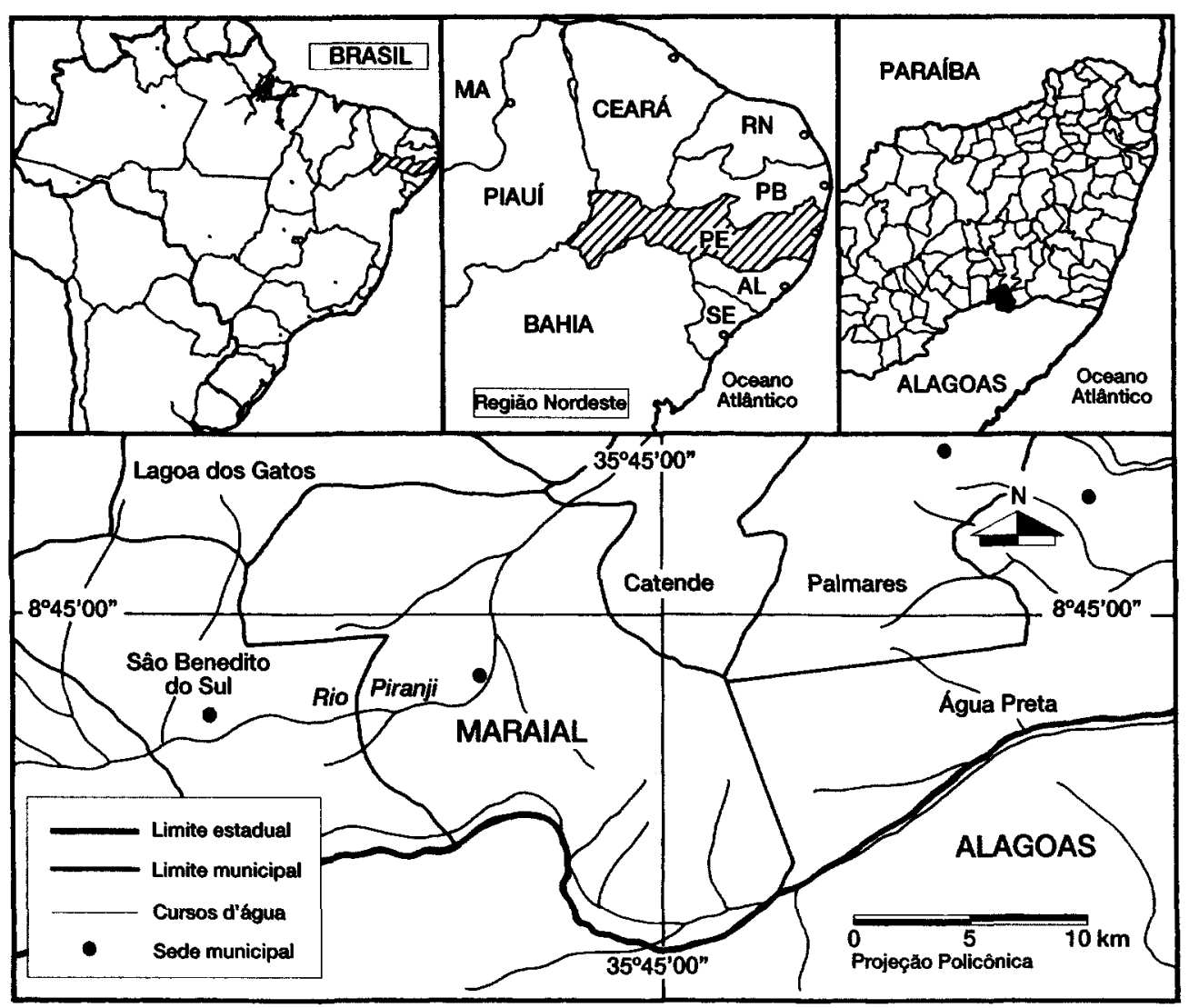

Fig. 1.-Map of the area. Maraial municipality, Forest South of the state, Pernambuco state, Brazil.

soil, with the exception of the Hemionitis palmata which was terrestrial on rocks and Vittaria lineata which is an epiphyte (table 1).

The proximity of sources of water and low light may affect species dominance, and main explain high number of species of Adiantum observed. Just six species, Lygodium venustum, L. volubile, Anemia villosa, Cyathea microdonta, Dicranopteris pectinata and $\mathrm{Pi}$ tyrogramma calomelanos, occur in sites subject open to high insolation, and generally affected by human activity. The rarest species found were Danaea elliptica and Hemionitis palmata, restricted to densely shaded and humid places. These species also present restricted distribution elsewhere in Pernambuco (BARROS, 1997).

\section{Marattiaceae}

\section{Danaea Sm.}

\subsection{D. elliptica Sm.}

Always terrestrial on soil, herbaceous, occasional in the forest, locally humid slopes, sciophilous.

Material examined: Mata do Ageró, 22-V-1998, M.R. Pietrobom-Silva 4337 \& al., PEUFR 25668.

\section{SchizaeaceaE}

\section{Lygodium Sw., nom. cons.}

\subsection{L. venustum $S w$.}

Terrestrial on soil, scandent, in ravines, heliophilous, not in groups; generally occurs at forest margins; frequent at collection site. 
TABLE 1

DISTRIBUTION OF PTERIDOPHYTES (MARATTIACEAE-VITTARIACEAE) WITH RESPECT TO HABITAT AND SUBSTRATE IN THE SERRA DO URUBú, MATA DO AgERó,

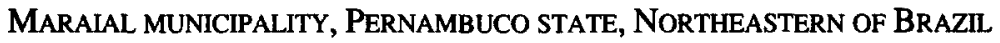

\begin{tabular}{|c|c|c|c|c|c|c|c|}
\hline \multirow[t]{2}{*}{ SPECIES } & \multicolumn{3}{|c|}{ HABITAT } & \multicolumn{4}{|c|}{ SUBSTRATUM } \\
\hline & Slopes & $\begin{array}{c}\text { Ravines/ } \\
\text { sun }\end{array}$ & $\begin{array}{l}\text { Ravines/ } \\
\text { shade }\end{array}$ & $\begin{array}{c}\text { Terrestrial } \\
\text { on soil }\end{array}$ & Epiphytics & $\begin{array}{c}\text { Terrestrial } \\
\text { on rocks }\end{array}$ & $\begin{array}{c}\text { Terrestrial } \\
\text { on soils } \\
\text { intermittent } \\
\text {-mind } \\
\text { flooded }\end{array}$ \\
\hline $\begin{array}{l}\text { MARATTIACEAE } \\
\text { Danaea elliptica }\end{array}$ & $x$ & 一 & - & $\times$ & - & - & - \\
\hline $\begin{array}{l}\text { SCHIZAEACEAE } \\
\text { Lygodium venustum } \\
\text { L. volubile } \\
\text { Schizaea elegans } \\
\text { Anemia villosa }\end{array}$ & $\begin{array}{l}- \\
x \\
-\end{array}$ & $\frac{x}{-}$ & $\frac{-}{x}$ & $\begin{array}{l}\times \\
\times \\
\times \\
\times\end{array}$ & $\begin{array}{l}- \\
- \\
-\end{array}$ & $\begin{array}{l}- \\
- \\
-\end{array}$ & $\begin{array}{l}- \\
- \\
-\end{array}$ \\
\hline $\begin{array}{l}\text { CYATHEACEAE } \\
\text { Alsophila pauciflora } \\
\text { Cyathea phalerata } \\
\text { C. microdonta }\end{array}$ & $\begin{array}{l}- \\
-\end{array}$ & $\frac{-}{x}$ & $\begin{array}{l}x \\
x \\
-\end{array}$ & $\begin{array}{l}x \\
x \\
x\end{array}$ & $\begin{array}{l}- \\
-\end{array}$ & - & - \\
\hline $\begin{array}{l}\text { GLEICHENIACEAE } \\
\text { Dicranopteris pectinata }\end{array}$ & - & $x$ & - & $x$ & - & - & - \\
\hline $\begin{array}{l}\text { PTERIDACEAE } \\
\text { Adiantopsis radiata } \\
\text { Adiantum dolosum } \\
\text { A. intermedium } \\
\text { A. glaucescens } \\
\text { A. macrophyllum } \\
\text { A. petiolatum } \\
\text { A. pulverulentum } \\
\text { A. pectinatum } \\
\text { A. terminatum } \\
\text { Hemionitis palmata } \\
\text { Pityrogramma calomelanos } \\
\text { Pteris denticulata }\end{array}$ & $\begin{array}{l}x \\
x \\
- \\
x \\
x \\
x \\
x \\
x \\
- \\
-\end{array}$ & $\begin{array}{l}- \\
- \\
- \\
- \\
- \\
- \\
- \\
-\end{array}$ & $\begin{array}{l}- \\
- \\
- \\
x \\
- \\
x \\
x \\
x \\
x \\
-\end{array}$ & $\begin{array}{l}\times \\
\times \\
\times \\
\times \\
\times \\
x \\
\times \\
\times \\
\times \\
- \\
\times \\
\times\end{array}$ & $\begin{array}{l}- \\
- \\
- \\
- \\
- \\
- \\
- \\
-\end{array}$ & $\begin{array}{l}- \\
- \\
- \\
- \\
- \\
- \\
- \\
-\end{array}$ & $\begin{array}{l}- \\
- \\
- \\
- \\
- \\
- \\
- \\
- \\
-\end{array}$ \\
\hline $\begin{array}{l}\text { VITTARIACEAE } \\
\text { Vittaria lineata }\end{array}$ & $x$ & - & - & - & $\times$ & - & - \\
\hline
\end{tabular}

Convention: $\times$ Present; - Absent.

Material examined: Mata do Ageró, 22-V-1998, M.R. Pietrobom-Silva 4340 \& al., PEUFR 25675.

1.2. L. volubile $S w$.

Terrestrial on soil, scandent, in ravines alongside highway, siophilous, not in groups; occasional.

Material examined: Mata do Agero, 22-V-1998, M.R. Pietrobom-Silva 4334 \& al.,PEUFR 25677. 
2. Anemia Sw., nom. cons.

\subsection{A. villosa Humb., Bonpl. ex Willd.}

Terrestrial on soil, in ravine alongside highway, growing in loamy soil, frequently heliophilous, in groups.

Material examined: Mata do Agero, 22-V-1998, J.A. Siqueira Filho, G.S. Baracho 793/7II, I.C.L. Barros, M.R. Pietrobom-Silva 4338 \& al., PEUFR 25674. 20IV-1994, A.M. Miranda 1573 \& L.P. Félix 120, PEUFR 16848.

3. Schizaea Sm., nom. cons.

\subsection{S. elegans (Vahl) Sm.}

Terrestrial on soil, along slopes within forest, sciohilous; rare.

Material examined: Mata do Ageró, 29-VIII-1987, L.P. Félix \& G.V. Dornelas, EAN 40101.

\section{Cyatheaceae}

1. Alsophila R. Br.

\subsection{A. pauciflora Kuhn}

Terrestrial on soil, in ravine alongside highway, sciophilous, not in groups; rare. First record for Pernambuco.

Material examined: Mata do Ageró, $900 \mathrm{~m}, 22-\mathrm{V}$ 1998, I.C.L. Barros, A.M. Moura Juinior, G.S. Baracho, J.A. Siqueira Filho, M.R. Pietrobom-Silva $4343 \&$ V. Gomes, PEUFR 25673.

\section{Cyathea Sm.}

\subsection{C. phalerata $M$. Martens}

Terrestrial on soil, in ravine alongside highway, sciophilous, not in groups; rare.

Material examined: Mata do Ageró, $900 \mathrm{~m}, 22-\mathrm{V}-$ 1998, I.C.L Barros, A.M. Moura Júnior, G.S. Baracho, J.A. Siqueira Filho, M.R. Pietrobom-Silva 4342 \& V. Gomes, PEUFR 25672.

\subsection{C. microdonta (Desv.) Domin}

Terrestrial on soil, on bank alongside highway, heliophilous, not in groups; rare.

Material examined: Mata do Ageró, $800 \mathrm{~m}, 13-\mathrm{III}-$ 1994, A.M. Miranda \& L.P. Félix 1454, PEUFR 16805.

\section{Gleicheniaceae}

\section{Dicranopteris Bernh.}

\subsection{D. pectinata (Willd.) Underw.}

Terrestrial on soil, in ravine alongside highway, heliophilous, not in groups; occasional.

Material examined: Mata do Ageró, $900 \mathrm{~m}, 22 \mathrm{~V}$. 1998, I.C.L. Barros, A.M. Moura Júnior, G.S. Baracho, J.A. Siqueira Filho, M.R. Pietrobom-Silva $434 I$ \& V. Gomes, PEUFR 25676.

\section{Ptermaceae Rchb.}

\section{Adiantopsis Fée}

\subsection{A. radiata (L.) Fée}

Terrestrial on soil, on banks alongside highway, sciophilous, not in groups; rare.

Material examined: Mata do Ageró, $900 \mathrm{~m}, 22-\mathrm{V}$ 1998, I.C.L. Barros 20, A.M. Moura Jünior, G.S. Baracho, J.A. Siqueira Filho, M.R. Pietrobom-Silva 4356 \& V. Gomes, PEUFR 25669.

\section{Adiantum L.}

\subsection{A. dolosum Kunze}

Terrestrial on soil, at margins of forest, alongside highway, sciophilous, not in groups; frequent.

Material examined: Mata do Ageró, $900 \mathrm{~m}, 22-\mathrm{V}$ 1998, I.C.L Barros, A.M. Moura Júnior, G.S. Baracho: J.A. Siqueira Filho, M.R. Pietrobom-Silva 4353 \& V. Gomes, PEUFR 25679. 29-VIII-1987, L.P. Félix 975, EAN 4118.

\subsection{A. intermedium Sw.}

Terrestrial on soil, in narrow ravine alongside highway, sciophilous, not in groups; frequent.

Material examined: Mata do Agero, $900 \mathrm{~m}, 22-\mathrm{V}$ 1998, I.C.L. Barros, A.M. Moura Júnior, G.S. Baracho, J.A. Siqueira Filho, M.R. Pietrobom-Silva 4348 \& V. Gomes, PEUFR 25684.

\subsection{A. glaucescens Klotzsch}

Terrestrial on soil, on slopes within forest, alongside highway, sciophilous, not in groups; frequent.

Material examined: Mata do Ageró, $900 \mathrm{~m}, 22-\mathrm{V}$ 1998, I.C.L Barros, A.M. Moura Júnior, G.S. Baracho, J.A. Siqueira Filho, M.R. Pietrobom-Silva 4345 \& V. 
Gomes, PEUFR 25687. 22-V-1998, I.C.L. Barros, A.M. Moura Juinior, G.S. Baracho, J.A. Siqueira Filho, M.R. Pietrobom-Silva 4347 \& V. Gomes, PEUFR 25682.

\subsection{A. macrophyllum $\mathrm{Sw}$.}

Terrestrial on soil, in narrow ravine alongside highway, sciophilous, not in groups; rare.

Material examined: Mata do Ageró, $900 \mathrm{~m}, 22-\mathrm{V}-$ 1998, I.C.L. Barros, A.M. Moura Júnior, G.S. Baracho, J.A. Siqueira Filho, M.R. Pietrobom-Silva 4354 \& V. Gomes, PEUFR 25680.

\subsection{A. petiolatum Desv.}

Terrestrial on soil, on slopes within forest, alongside highway, sciophilous, not in groups; occasional.

Material examined: Mata do Ageró, $900 \mathrm{~m}, 22-\mathrm{V}-$ 1998, I.C.L. Barros, A.M. Moura Júnior, G.S. Baracho, J.A. Siqueira Filho, M.R. Pietrobom-Silva $4349 \&$ V. Gomes, PEUFR 25683.

\subsection{A. pulverulentum $L$.}

Terrestrial on soil, on slopes and closed ravines alongside highway, sciophilous, not in groups; frequent.

Material examined: Mata do Ageró, $900 \mathrm{~m}, 22-\mathrm{V}$ 1998, I.C.L. Barros, A.M. Moura Júnior, G.S. Baracho, J.A. Siqueira Filho, M.R. Pietrobom-Silva 4346 \& V. Gomes, PEUFR 25681.

\subsection{A. terminatum Kunze ex Miq.}

Terrestrial on soil, at margins of forest and in closed ravines alongside highway, sciophilous, not in groups; frequent.

Material examined: Mata do Ageró, $900 \mathrm{~m}, 22-\mathrm{V}$ 1998, I.C.L. Barros, A.M. Moura Júnior, G.S. Baracho, J.A. Siqueira Filho, M.R. Pietrobom-Silva 4352 \& V. Gomes, PEUFR 25686. 22-V-1998, I.C.L. Barros, A.M. Moura Júnior, G.S. Baracho, J.A. Siqueira Filho, M.R. Pietrobom-Silva 4351 \& V. Gomes, PEUFR 25685. 13III-1994, A.M. Miranda $1418 \&$ L.P. Félix, PEUFR 15950.

\subsection{A. pectinatum Kunze}

Terrestrial on soil, at margins of forest, sciophilous, not in groups; frequent.

Material examined: Mata do Ageró, $900 \mathrm{~m}, 29-\mathrm{VIII}-$ 1987, L.P. Félix 1000 \& G.V. Dornelles, EAN 4089.

\section{Hemionitis L.}

\subsection{H. palmata $L$.}

Terrestrial on rocks, within forest, sciophilous, grouped; rare.

Material examined: Mata do Ageró, $900 \mathrm{~m}, 22-\mathrm{V}$ 1998, I.C.L. Barros, A.M. Moura Júnior, G.S. Baracho, J.A. Siqueira Filho, M.R. Pietrobom-Silva $4357 \&$ V. Gomes, PEUFR 25670.

\section{Pityrogramma Link}

\subsection{P. calomelanos (L.) Link}

Terrestrial on soils alongside irrigation ditches, heliophilous, not in groups; occasional.

Material examined: Mata do Agero, $900 \mathrm{~m}, 22-\mathrm{V}-$ 1998, I.C.L. Barros, A.M. Moura Júnior, G.S. Baracho, J.A. Siqueira Filho, M.R. Pietrobom-Silva 4358 \& V. Gomes, PEUFR 25666.

\section{Pteris L.}

\subsection{P. denticulata $S w$.}

Terrestrial on soil, on banks alonside highway, sciophilous, not in groups; rare.

Material examined: Mata do Ageró, $900 \mathrm{~m}, 22-\mathrm{V}$ 1998, I.C.L. Barros, A.M. Moura Junior, G.S. Baracho, J.A. Siqueira Filho, M.R. Pietrobom-Silva 4344 \& V. Gomes, PEUFR 25678.

\section{VITTARIACEAE}

\section{Vittaria J. Sm.}

\subsection{V. lineata (L.) Sm.}

Epiphytic holocorticicolous, on logs in decomposition, on banks of forest, sciophilous, associated with briophytes; rare.

Material examined: Mata do Ageró, $900 \mathrm{~m}, 22-\mathrm{V}$ 1998, J.A. Siqueira-Filho \& G.S. Baracho 794/718, I.C.L. Barros, M.R. Pietrobom-Silva 4359 \& V. Gomes, PEUFR 25667.

\section{REFERENCES}

Ambrósio, S.T. (1995). Pteridofitas da Reserva Ecológica de Jangadinha - Jaboatão dos Guararapes - Pernambuco. Recife [Dissertação de Mestrado. Curso de Mestrado em Criptógamos. Departamento de Botânica, Departamento de Micologia, Universidade Federal de Pernambuco].

Ambrósio, S.T. \& I.C.L. Barros (1997). Pteridófitas de uma área remanescente de Floresta Atlântica do estado 
de Pemambuco, Brasil. Acta Botabica Brasilica 11: 105-113.

ANDRADE-LimA, D. (1960). Estudos Fitogeográficos de Pernambuco. Arquivos do Instituto de Pesquisas Agronômicas 5: 305-341.

BARROS, I.C.L. (1980). Taxonomia, fitogeografia e morfologia das Schizaeaceae do Nordeste Brasileiro. Recife, 1980. Dissertação. (Mestrado em Botânica). Departamento de Biologia, Universidade Federal Rural de Pernambuco.

Barros, I.C.L. (1996). As Pteridófitas. In: E.V.S.B. Sampaio, S.J. Mayo \& M.R.V. Barbosa, Pesquisa Botânica Nordestina - Progresso e Perspectivas, Ed. Universitária/UFPE, Recife, pp. 111-121. Recife.

Barros, I.C.L. (1997). Pteridófitas ocorrentes em Pernambuco: Ensaio Biogeográfico e Análise Numérica. Recife, 1997. 557 f. [Tese de Doutorado. Programa de Pós-Graduação em Botânica. Departamento de Biologia, Universidade Federal Rural de Pernambuco].

BARROS, I.C.L. (1998). Biodiversidade e Ecologia das espécies de pteridófitas (avencas, samambaias e plantas afins) da Reserva Ecológica de Dois Irmãos. In: $R e$ serva Ecológica de Dois Irmãos: Estudos em um re. manescente de Mata Atlântica em área urbana (Recife - Pernambuco - Brasil). Ed. Governo do Estado de Pernambuco, Secretaria de Ciência, Tecnologia e Meio Ambiente - SECTMA. Ed. Universitária/UFPE, Recife, pp. 136-156.

BarRos, I.C.L. \& G. MARIZ (1990). Novas Referências de pteridófitas para Pernambuco (BR). Anais do XXXV Congresso Nacional de Botânica (1985), Curitiba, $P R$, pp. 201-229. Brasilia.

BARROS, I.C.L. \& al. (1988). Distribuição geográfica das pteridófitas ocorrentes no estado de Pernambuco, Brasil. Acta Botanica Brasílica. Porto Alegre, 2, $(1,2)$ : 47-84.

BARros, I.C.L. \& al. (1989). Adições à Flora Pteridofítica do estado de Pernambuco. Biologica Brasilica, Recife, 1(1): 79-83.

BARROS. I.C.L. \& al. (1992a). Novas Referências de pteridófitas para Pemambuco II. Pteridaceae. Dennstaedtiaceae. Thelypteridaceae. Dryopteridaceae. Anais do XXXVI Congresso Nacional de Botanica (1986), Ouro Preto, $M G$, pp. 28-72. Belo Horizonte.

BarRos, I.C.L. \& al. (1992b). Novas Referências de pteridofitas para Pernambuco III. Aspleniaceae. Davallaceae. Polypodiaceae. Marsileaceae. Anais do XXXVI Congresso Nacional de Botânica (1986), Ouro Preto, MG, pp. 73-116. Belo Horizonte.

BARRos, I.C.L. \& al. (1992c). Nova Referência de pteridófita em área remanescente da Floresta Atlântica (Mata de Dois Irmãos - Recife - PE). Anais do XXXVI Congresso Nacional de Botânica (1986), Ouro Preto, $M G$, pp. 201-229. Belo Horizonte.

BARROS, I.C.L. \& al. (1992d). Ocorrência de Asplenium pumilum Sw. na Serra dos Ventos - Belo Jardim (PE). Biologica Brasilica 4(1/2): 23-82.

BARROS, I.C.L. \& al. (1997a). Contribuição ao estudo taxonômico das pteridófitas ocorrentes na Reserva Ecológica de Caetés (Paulista-PE). Thelypteridaceae. Dryopteridaceae. Davalliaceae. Polypodiaceae. Lyco- podiaceae. Boletim da Sociedade Broteriana ser. 2, 67: 271-286.

BARROS, I.C.L. \& al. (1997b). Lycopodiaceae Myrbel de Brejo dos Cavalos, Caruarú - Pernambuco. Boletim da Sociedade Broteriana ser. 2, 67: 263-269.

BARROS, I.C.L. \& al. (1997c). Taxonomia, padrão de venação e aparelhos estomátıcos de Pteris schwakeana Chris. (Pteridophyta, Pteridaceae). Boletim da Sociedade Brotereiana, ser. 2, 67: 257-262.

BRUMMIT, R.K. \& PowELL, C.E. (1992). Authors of Plant Names. Royal Botanical Garden, Kew.

CONDEPE - Instituto de Planejamento de PernamBUCO (1995). Anuário Estatístico de Pernambuco (1992) 1: 19-32.

FARIAS, M.C.A. \& al. (1992). Pteridófitas da Reserva de Caetés (Paulista - PE). Boletim da Sociedade Broteriana, ser. 2, 65: 149-162.

Ferreira, M.F.A., M.J.N. Rodal \& G.H. Caryalho (1985). Vegetação de Pernambuco. Anais da VIII Reunião Nordestina de Botânica 1: 245-249.

FonSECA, E.R. (1992). Pteridófitas da Reserva do Gurjaú, Cabo, Pernambuco. Recife [Dissertação de Mestrado, Curso de Mestrado em Criptógamos. Departamento de Botânica, Departamento de Micologia, Universidade Federal de Pernambuco].

Holmgren, P.K., N.H. Holmgren, \& L.C. BarnetT (1990). Index Herbariorum, Part I: The Herbaria of the World, ed. 8. Inst. Ass. Plant Taxonomy / New York Botanical Garden, New York.

LELLINGER, D.B. (1987). The disposition of Trichopteris (Cyatheaceae). American Fern Journal 77(3): 90-94.

LuetzelburG, P. (1923). Estudo Botanico do Nordeste. Inspetoria Federal de Obras Contra as Secas. Rio de Janeiro, Ser. 1, 2(57): 1-283.

Pontual, I.B. (1969). Pteridofitas de Pernambuco e Alagoas (I). Anais do XXI Congresso Nacional de Botânica, Goiânia, Sociedade Botânica do Brasil: 185-192.

PontuAl, I.B. (1971). Pterid6fitas de Pernambuco e Alagoas (II). Anais do Instituto de Biologia, Recife, 1(1): 153-260.

PonTUAL, I.B. (1972). Pteridófitas do Nordeste. Anais do XXIII Congresso Nacional de Botânica, Garanhúns, Sociedade Botânica do Brasil: 69-72.

SEHNEM, A. (1967). Marattiáceas. In: R. Reitz. (ed.), Flora llustrada Catarinense. Itajaí, p. 1-68.

SEHNEM, A. (1968). Aspleniáceas. In: R. Reitz. (ed.), Flora llustrada Catarinense. Itajaí, p. 1-173.

SEHNEM, A. (1970). Gleiqueniáceas. In: R. Reitz. (ed.), Flora llustrada Catarinense. Itajaí, p. 158.

SEHNEM, A. (1972). Pteridáceas. In: R. Reitz. (ed.), Flora Ilustrada Catarinense. Itajaí, p. 1-244.

SEHNEM, A. (1974). Esquizeáceas. In: R. Reitz. (ed.), Flora llustrada Catarinense. Itajaí, p. 178.

SEHNEM, A. (1978). Cíateáceas. In: R. Reitz. (ed.), Flora Ilustrada Catarinense. Itajaí, p. 1-115

TryoN, R.M. \& R.G. STOLze (1989a). Pteridophyta of Peru. Part I. 1. Ophioglossaceae - 12. Cyatheaceae. Fieldiana Botany, New Series, 21: 1-120, Chicago.

Tryon, R.M. \& R.G. Stolze (1989b). Pteridophyta of Peru. Part II. 13. Pteridaceae - 15. Dennstaedtiaceae. Fieldiana Botany, New Series, 22: 1-128, Chicago. 
TrYon, R.M. \& A.F. TrYoN (1982). Ferns and Allied Plants, with special reference to Tropical America. Springer Verlag, New York, 857 p.

Veloso, H.P. \& L. Goes Filho (1982). Fitogeografia brasileira, classificação fisionômico - ecológica da vegetação neotropical. Boletim Técnico Projeto RADAMBRASIL. Série Vegetação, Salvador 1: 1-80. Windisch, P.G. (1983). Pteridófitas da Serra Ricardo Franco (Estado de Mato Grosso) e aspectos dos seus macrohabitates. São José do Rio Preto [Tese de Li- vre Docência. Departamento de Botânica, Universidade Estadual Paulista "Júlio de Mesquita Filho", UNESP].

Windisch, P.G. (1992). Pteridófitas da Regiäo NorteOcidental do Estado de São Paulo: Guia para estudo e excursões, ed. 2. São José do Rio Preto.

Editado por Félix Muñoz Garmendia Aceptado para publicación: 25-VIII-2000 